Published in "SIAM Journal on Applied Mathematics 68(3): 869-889, 2008"

which should be cited to refer to this work.

\title{
A MATHEMATICAL MODEL FOR THE STEADY ACTIVATION OF A SKELETAL MUSCLE*
}

\author{
J.-P. GABRIEL ${ }^{\dagger}$, L. M. STUDER ${ }^{\ddagger}$, D. G. RÜEGG ${ }^{\S}$, AND M.-A. SCHNETZER
}

\begin{abstract}
A skeletal muscle is composed of motor units, each consisting of a motoneuron and the muscle fibers it innervates. The input to the motor units is formed of electrical signals coming from higher motor centers and propagated to the motoneurons along a network of nerve fibers. Because of its complexity, this network still escapes actual direct observations. The present model describes the steady state activation of a muscle, i.e., of its motor units. It incorporates the network as an unknown quantity and, given the latter, predicts the input-force relation (activation curve) of the muscle. Conversely, given a suitable activation curve, our model enables the recovery of the network. This step is performed by using experimental data about the activation curve, and the whole activation process of a muscle can then be theoretically investigated. In this way, this approach provides a link between the macroscopic (activation curve) and microscopic (network) levels. From a mathematical viewpoint, solving the preceding inverse problem is equivalent to solving an integral equation of a new type.
\end{abstract}

1. Introduction. The activation of a muscle is a fascinating phenomenon involving complex and subtle physiological processes. Muscles responsible for voluntary motions are called striated (or skeletal) and are composed of motor units (MUs) consisting of a motoneuron (MN) together with the muscle fibers under its control. Depending on the muscle, the number of MUs can vary from ten up to several thousands. The MNs are located in the spinal chord and are connected to the central nervous system (CNS) through nerve fibers (input fibers) propagating the signal (input) in the form of electrical impulses called action potentials (APs).

When an AP reaches an MN through a (synaptic) contact, it modifies the electrical potential of its membrane, generating a so-called excitatory postsynaptic potential (EPSP). On a given MN, the effects of different APs are supposed to be additive. When the membrane potential reaches a specific threshold value, the MN starts generating APs which are transmitted to the muscle fibers and induce their contractions. As the activity of the input fibers increases, new stronger MUs are recruited (size principle). Additionally already active MUs enhance their forces, a process called frequency modulation $[8,9]$. As soon as all MUs are recruited, frequency modulation is the only way for a muscle to increase its force. For simplicity reasons, we consider here only stationary isometric contractions and we assume that the total muscle force

\footnotetext{
${ }^{\dagger}$ Department of Mathematics, University of Fribourg, Rue du Musée 5, 1700 Fribourg, Switzerland (jean-pierre.gabriel@unifr.ch).

${ }^{\ddagger}$ Department of Computer Science and Economics, University of Applied Sciences, av. Max-Huber 6, 3960 Sierre, Switzerland (leo.studer@mus.ch).

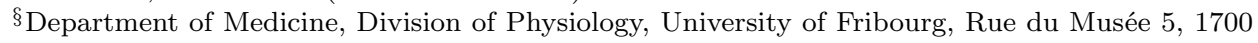
Fribourg, Switzerland (dieter.ruegg@unifr.ch).

『 Department of Mathematics, University of Applied Sciences, bd de Pérolles 82, 1700 Fribourg, Switzerland (marc-adrien.schnetzer@hefr.ch).
} 
is given by the sum of all MU forces. The input-force relation of a muscle or its graph is alternatively called the activation curve.

MUs are ordered according to their maximal (tetanic) contraction forces $t$. The way input fibers activate MNs is extremely complex and the details of a corresponding network are not yet understood. On the level of an MN, this network, also called synaptic weight and denoted $g(t)$ (or sometimes $g$ ), is the missing quantity in our approach. Observations provide information about the shape of an activation curve. Our model is then used to recover $g$ from this information (inverse problem). At this point, all aspects of the activation process of a muscle can be predicted.

For an arbitrary but fixed network, let $F(t)$ be the muscle force as a function of the last recruited MU. Clearly $F(t)$ has to be an increasing function of $t$. It will be seen that $g(t)$ can be deduced directly from $F(t)$ and it is thus sufficient to focus on the last function. $F(t)$ turns out to be a solution of an integral equation of the form

$$
F(t)=\int_{a}^{t} k(s, F(s), F(t)) d s,
$$

and our task will be to solve (1). The presence of $F(t)$ in the kernel of (1) has striking consequences: on one hand this integral equation is not a classical Volterra equation and thus belongs to an extended type; on the other hand it admits infinitely many discontinuous solutions. Fortunately this equation has a unique continuous solution and this property turns out to be equivalent to increasingness. Since $F(t)$ is increasing in $t,(1)$ admits one and only one physiologically meaningful solution. By using the latter, (1) can be reduced to a classical Volterra equation whose analytical and numerical properties are well known.

Analysis shows that the activation process has the following interesting properties:

(a) Similarly to the notion of the standard normal random variable in probability theory, there exists a standard hyperbolic muscle from which the activation curve of any muscle can be obtained.

(b) Despite the large number of parameters involved in the model, any activation curve is totally determined by a unique number called the activation factor.

(c) Several functionals related to the activation process can be introduced and characterized analytically, e.g., the ratio of the forces due to frequency modulation and recruitment.

All the published models of the MN pool-muscle complex (MNPMC) quantitatively describe the relation between the input to the MN pool and the muscle force $[17,18,43,12]$ or the electromyogram [12]. The models are similar in their structure but differ in the choice of the quantities given a priori. In the literature, all MNPMC models have been reduced to the following three unknowns: (1) the synaptic weight, (2) the MU population, and (3) the activation curve or the electromyogram. Since two of the unknowns can be given and the third one can be deduced from the model, we have three possible configurations: (1) and (2) are given and (3) is computed [17, 12]; (1) and (3) are given and (2) is computed [43]; and - the approach that we proposethe MU population (2) and the activation curve (3) are given and the synaptic weight (1) is determined. The main reason for this choice is that data at the level of the MNs are available for both unknowns (2) and (3) but not for unknown (1). As mentioned above, this configuration also leads to a new and interesting mathematical problem.

1.1. The model. We expose here the mathematical aspects of the model developed in [42], where a thorough discussion of the physiological hypotheses can be found. We focus mainly on equation (1), which is the key to the present investigation. 
Since a typical skeletal muscle contains several hundred MUs, it is adequate to represent the MU population by a density function $\rho$. Choosing the tetanic contraction force $t$ as variable, we get $t \in\left[t_{\min }, t_{\max }\right] \mapsto \rho(t)$, where $t_{\min }$ and $t_{\max }$ are the tetanic forces of the weakest and strongest MUs and the number of MUs in the pool is given by $\int_{t_{\min }}^{t_{\max }} \rho(s) d s$. All our considerations hold for all integrable and almost everywhere (a.e.) strictly positive functions $\rho$. We assume that $t_{\min }$ and $t_{\max }$ are given through $\rho$.

The global input In to the MNPMC is defined as the sum of all AP frequencies [43]. Each fiber contacts each MN of the pool and its activity induces EPSP conductance changes in the postsynaptic membrane. The EPSPs generated by single input fibers are smaller than $100 \mu \mathrm{V}$ [15], the activity of these fibers is asynchronous [7], and the voltage threshold for APs is about $12 \mathrm{mV}$ [5]. These three experimental findings imply that the variations of the membrane potential are smaller than $1 \%$ of the threshold voltage and are therefore neglected in the model. The EPSP conductances are thus represented by their time averages in the present time-independent approach. Due to the lack of precise information and for simplicity reasons, we assume that the EPSP conductance $G_{E P S P}$ of each MN is proportional to the input:

$$
G_{E P S P}(I n)=g I n,
$$

where the MN-dependent factor $g$ is the synaptic weight. This linearity assumption entails some restrictions on the MN connectivity [42].

The inactive (or subthreshold) MN is modeled with a single compartment and a homogeneous, electrically isolated membrane obeying Ohm's law. The total membrane current $i_{\text {tot }}$ is the sum of the capacitive and ionic currents:

$$
i_{\text {tot }}=C \dot{U}+\sum_{k} G_{k}\left(U-E_{k}\right)
$$

where $U$ is the membrane potential, $\dot{U}$ the time derivative of $U, G_{k}$ the conductance of ion $k, E_{k}$ its equilibrium potential, and $C$ the membrane capacity. Three types of ionic conductances are distinguished: (a) a transmitter-sensitive conductance $G_{k}^{E}$ caused by the synaptic input, (b) a voltage-dependent conductance $G_{k}^{U}$ generating APs and $E_{k}^{U}$ its equilibrium potential, and (c) a leakage conductance $G_{L}$ and $E_{L}$ its equilibrium potential. Clearly, we have $G_{E P S P}=\sum_{k} G_{k}^{E}$. For lack of data about particular MNs that are activated by synaptic input, we rely on data from current injection experiments [24]. The capacitive current is 0 in the steady state and since the membrane is isolated, the total current is equal to the injected current $i_{i n j}$. Consequently, (3) can be rewritten $i_{i n j}=\sum_{k} G_{k}^{E}\left(U-E_{k}\right)+\sum_{k} G_{k}^{U}\left(U-E_{k}^{U}\right)+$ $G_{L}\left(U-E_{L}\right)=G_{E P S P}\left(U-\frac{\sum_{k} G_{k}^{E} E_{k}}{G_{E P S P}}\right)+\sum_{k} G_{k}^{U}\left(U-E_{k}^{U}\right)+G_{L}\left(U-E_{L}\right)$. Introducing the variable $V:=U-E_{L}$, the reversal potential of the EPSP current $E_{E P S P}:=$ $\frac{\sum_{k} G_{k}^{E} E_{k}}{G_{E P S P}}-E_{L}$, and $E_{k}^{V}:=E_{k}^{U}-E_{L}$, we then get

$$
i_{i n j}=G_{E P S P}\left(V-E_{E P S P}\right)+\sum_{k} G_{k}^{U}\left(V-E_{k}^{V}\right)+G_{L} V .
$$

The current $i_{E P S P}:=G_{E P S P}\left(V-E_{E P S P}\right)$ will be called the EPSP induced current.

A subthreshold $\mathrm{MN}$ is inactive as long as it receives synaptic inputs without generating APs. During subthreshold depolarizations, there is a small increase of the sodium conductance which tends to depolarize the membrane and a small increase of the potassium conductance which tends to hyperpolarize the membrane. Since the 
two currents are in opposite directions, they tend to cancel each other (ultimately, this could be quantitatively derived from the Hodgkin-Huxley equations [22]). Therefore, we assume that the voltage-dependent channels are closed in subthreshold MNs, and consequently, the voltage-dependent currents are 0 . Moreover, if $i_{i n j}=0$, the membrane potential corresponding to the EPSP is denoted $V_{E P S P}$ and (4) becomes

$$
G_{E P S P}\left(V_{E P S P}-E_{E P S P}\right)+G_{L} V_{E P S P}=0 .
$$

According to (2) and (5), the membrane potential as a function of the input is

$$
V_{E P S P}(I n)=\frac{g I n E_{E P S P}}{G_{L}+g I n} .
$$

The threshold input $I n_{T}$ is the maximum input an MN can receive in the subthreshold state. It evokes an EPSP equal to the firing threshold voltage $V_{T}$ and therefore satisfies $V_{E P S P}\left(I n_{T}\right)=V_{T}$. Assuming that all MNs of the pool have the same firing threshold [42], (6) at threshold provides

$$
\tilde{g}=\frac{V_{T}}{\left(E_{E P S P}-V_{T}\right) I n_{T}},
$$

where $\tilde{g}:=\frac{g}{G_{L}}$ is called relative synaptic weight. Two quantities in (7) are MUdependent, namely, the relative synaptic weight and the threshold input. According to $(7)$, it is equally adequate to determine either one of them.

Fitting the frequency-injected current relations with a straight line with slope $\kappa$ $[42,24]$, the MN firing frequency $\nu\left(G_{E P S P}, i_{i n j}\right)$ as a function of $G_{E P S P}$ and $i_{i n j}$ is given by

$$
\nu\left(0, i_{i n j}\right)=\kappa\left(i_{i n j}-i_{T}\right)+\nu_{T} \text { if } i_{i n j} \geq i_{T} \text { and } 0 \text { otherwise }
$$

where $\nu_{T}$ is the threshold frequency and $i_{T}$ the threshold current. Estimations of their values have been determined with current injection experiments on MNs [25].

The relation between the frequency evoked by a synaptic input and the injected current is not at all simple since $i_{E P S P}$, but not $i_{i n j}$, depends on the membrane potential. We look for an injected current $i_{i n j}\left(G_{E P S P}\right)$ which evokes the same frequency as the synaptic input, namely, $\nu\left(G_{E P S P}, 0\right)=\nu\left(0, i_{i n j}\left(G_{E P S P}\right)\right)$.

Let us suppose that such a current exists for all values of $G_{E P S P}: i_{i n j}\left(G_{E P S P}\right)=$ $-i_{E P S P}$. Although the membrane potential of active MNs varies, we replace it by a constant virtual potential $V_{A}$ which is independent of the firing frequency and is supposed to have similar effects as the time-varying membrane potential. In this approach, we set $V_{A}=V_{T}$ and we get $i_{i n j}\left(G_{E P S P}\right)=G_{E P S P}\left(E_{E P S P}-V_{T}\right)$. By inserting (2) and (7) into the last equation, we obtain $i_{i n j}\left(G_{E P S P}(\operatorname{In})\right)=\frac{G_{L} V_{T} I n}{I n_{T}}$. Since $I n=I n_{T}$ at threshold, the injected threshold current is $i_{T}=G_{L} V_{T}$.

The frequency-force relation of an MU during maintained contractions is built by fitting data obtained by injecting long-lasting currents of different intensities into MNs [26]. The MU force is given by

$$
f(\nu)=t\left(1-c \exp \left(-\gamma\left(\nu-\nu_{T}\right)\right)\right)
$$

where $t$ is the MU tetanic contraction force, $\nu_{T}$ is its threshold frequency, and $\gamma$ controls the shape of the curve. The number $c$ determines the fraction of the tetanic force at recruitment according to $f\left(\nu_{T}\right)=t(1-c)$. Since MUs are parameterized 
by their tetanic contraction forces $t$, the threshold input $I n_{T}(t)$ is the smallest value necessary to recruit the $\mathrm{MU}$ with tetanic force $t$. Equation (8), $i_{T}=G_{L} V_{T}$, and (9) lead to the following representation of the MU transfer function:

$$
f(t, I n):=t\left(1-c \exp \left(-\alpha \frac{\operatorname{In}-\operatorname{In}_{T}(t)}{\operatorname{In}(t)}\right)\right) \text { if } \operatorname{In}>\operatorname{In}(t) \text { and } 0 \text { otherwise, }
$$

where $\alpha:=\gamma \kappa G_{L} V_{T}$.

In muscles with parallel fibers, the total muscle force is the sum of the contraction forces of its MUs, and thus its activation curve is $\mathcal{F}(\operatorname{In})=\int_{t_{\text {min }}}^{t_{\max }} \rho(s) f(s, I n) d s$. Figure 1 depicts the different steps leading from In to $\mathcal{F}(\operatorname{In})$.

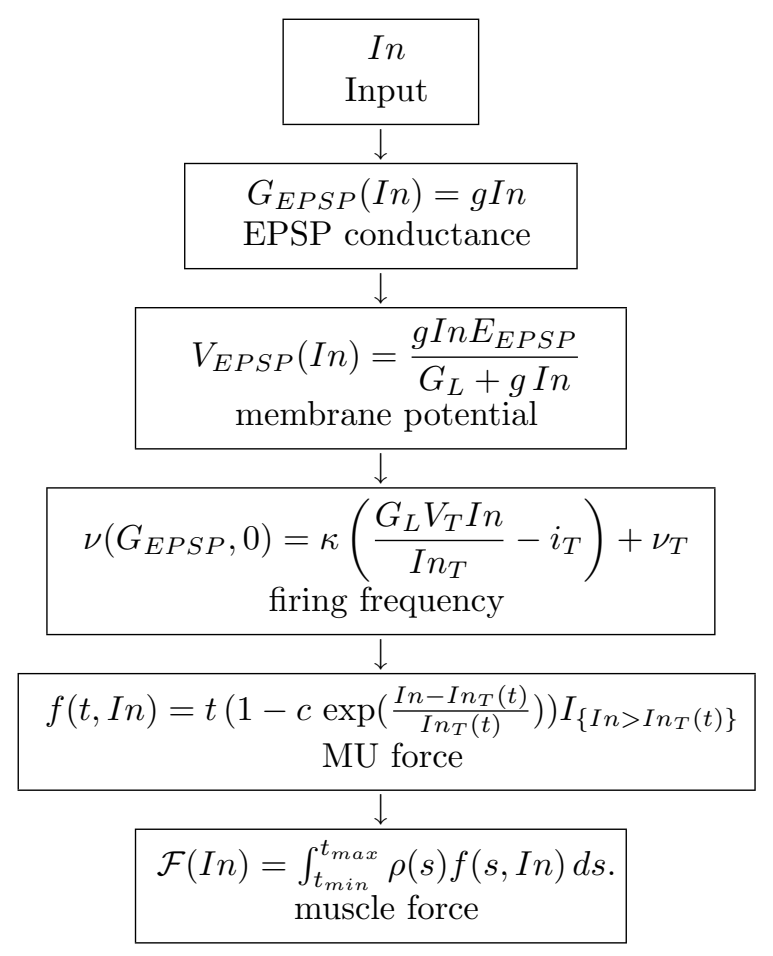

FIG. 1. Steps for the construction of the activation curve $\mathcal{F}($ In $)$.

According to the size principle [21], MUs are recruited according to their tetanic contraction force during muscle activation; i.e., weaker MUs are recruited before stronger MUs. Therefore, the threshold input $\operatorname{In}_{T}(t)$ activates all MUs with tetanic forces smaller than or equal to $t$. Replacing $I n$ by $\operatorname{In}_{T}(t)$, we obtain

$$
\mathcal{F}\left(\operatorname{In}_{T}(t)\right)=\int_{t_{\text {min }}}^{t} \rho(s) f\left(s, \operatorname{In}_{T}(t)\right) d s .
$$

Human subjects who superimposed ballistic contractions on background activities of different levels [38] provided information to determine the unknown function $\operatorname{In}_{T}(t)$. The data suggest that the force generated by two inputs $I n_{1}$ and $I n_{2}$ is the sum of the forces induced by the single inputs. If $I n_{0}$ denotes the minimal input necessary to recruit the smallest $\mathrm{MU}$ of the pool, we get the functional equation $\mathcal{F}\left(I n_{0}+I n_{1}+I n_{2}\right)=$ $\mathcal{F}\left(I n_{0}+I n_{1}\right)+\mathcal{F}\left(I n_{0}+I n_{2}\right)$. Its unique nonnegative solution with $\mathcal{F}\left(I n_{0}\right)=0$ is 
(see [1]) $\mathcal{F}(\operatorname{In})=k\left(\operatorname{In}-\operatorname{In}_{0}\right)$. This is the equation of a straight line with slope $k$. After recruitment, the activation curve is strictly concave. Since no additional force due to newly recruited MUs is available, it is an integral of strictly concave functions given in (10). Consequently, the affine ("linear" in [42]) relation can hold exclusively during recruitment. In a forthcoming paper, we will show that the possible activation curves are not limited to affine functions.

It should also be noted that the value of In corresponding to the end of recruitment is not specified a priori. This is a "free boundary" which will be determined by the model.

One could wonder whether a muscle response, which is not exactly additive, can be reasonably approximated by an additive function or not. The following results [29] shed some light on this question: a function $f: \mathbb{R} \rightarrow \mathbb{R}$ is called $\epsilon$-additive if $|f(x+y)-f(x)-f(y)| \leq \epsilon$ for all $x, y \in \mathbb{R}$. It can be seen that, if $g$ is additive, any function $f$ fulfilling $|f(x)-g(x)| \leq \epsilon$ for all $x \in \mathbb{R}$ is $3 \epsilon$-additive. Conversely, it can be proved that, for any $\epsilon$-additive function $f$, there exists a unique additive function $g: \mathbb{R} \rightarrow \mathbb{R}$ such that $|f(x)-g(x)| \leq \epsilon$, given by $g(x)=\lim _{n \rightarrow \infty} \frac{f(n x)}{n}$. As a consequence, if a muscle response $\mathcal{F}$ is only $\epsilon$-additive, then there exists an additive function $g$ contained in a band of width $2 \epsilon$ around $\mathcal{F}$. Moreover, if $\mathcal{F}$ is either bounded above or measurable, then $g$ is continuous.

Replacing $I n$ by $I n_{T}(t)$ as above, we get, for $t \in\left[t_{\text {min }}, t_{\text {max }}\right], \mathcal{F}\left(\operatorname{In}_{T}(t)\right)=$ $k\left(\operatorname{In}_{T}(t)-I n_{0}\right)$, and with (10) and (11),

$$
\mathcal{F}\left(\operatorname{In}_{T}(t)\right)=\int_{t_{\text {min }}}^{t} h(s)\left(1-c \exp \left(-\alpha \frac{\mathcal{F}\left(\operatorname{In}_{T}(t)\right)-\mathcal{F}\left(\operatorname{In}_{T}(s)\right)}{\mathcal{F}\left(\operatorname{In}_{T}(s)\right)+\Delta}\right)\right) d s,
$$

where $h(t)=t \rho(t)$ is the force density function of the muscle and $\Delta=k \operatorname{In} n_{0}$.

Equation (12) contains the parameters $\alpha, c, \Delta$, and $h$ [42]. Experimental observations suggest that $\alpha[24,26,2]$ and $c$ [27] are MU-independent, and, in the present approach, we assume muscle independence. On the basis of experimental data, $\alpha$ was set to 1.14 and $c$ to 0.9 [42], but the forthcoming general discussion is valid for all $\alpha>0,0<c<1$, and $\Delta>0$. A muscle is thus specified by $\Delta$ and $h$. $I n_{0}$ cannot be measured experimentally, and in [42] it was assumed to be the same for all muscles. This assumption is however not required here.

\section{The integral equation.}

2.1. General considerations. Equation (12) is an integral equation for the unknown function $\mathcal{F}\left(\operatorname{In}_{T}(t)\right)$. By introducing the notation $F(t)=\mathcal{F}\left(\operatorname{In}_{T}(t)\right),(12)$ takes the form

$$
F(t)=\int_{t_{\text {min }}}^{t} h(s)\left(1-c \exp \left(-\alpha \frac{F(t)-F(s)}{F(s)+\Delta}\right)\right) d s, \quad t \in\left[t_{\min }, t_{\max }\right] .
$$

With our assumptions about $\rho$, the preceding integral has to be understood in the Lebesgue sense and, for obvious physiological reasons, we look for nonnegative solutions.

Equation (13) is not of Volterra type because its kernel

$$
k(s, F(s), F(t)):=h(s)\left(1-c \exp \left(-\alpha \frac{F(t)-F(s)}{F(s)+\Delta}\right)\right)
$$

involves $F(t)$ and not only $s, t$, and $F(s)$. If (13) has a nonnegative locally bounded solution $F(t), t \in\left[t_{\min }, t_{\max }\right]$, for $\alpha>0,0<c<1$, and $\Delta>0$, it may admit discontinuous solutions. Indeed, as a consequence of the dominated convergence theorem, for 
every sequence $\left(t_{n}\right)_{n \in \mathbb{N}}, t_{n} \in\left[t_{\text {min }}, t_{\text {max }}\right]$, with $\lim _{n \rightarrow \infty} t_{n}=t$ and $\lim _{n \rightarrow \infty} F\left(t_{n}\right)=\gamma$, we have $\lim _{n \rightarrow \infty} \int_{t_{\text {min }}}^{t_{n}} k\left(s, F(s), F\left(t_{n}\right)\right) d s=\int_{t_{\text {min }}}^{t} k(s, F(s), \gamma) d s$. Thus (13) admits a locally bounded solution discontinuous at $t^{*} \in\left[t_{\min }, t_{\max }\right]$ if and only if the set $A_{t^{*}}=\left\{x \in \mathbb{R} ; x=\int_{t_{\text {min }}}^{t^{*}} k(s, F(s), x) d s\right\}$ contains an element $\beta \neq F\left(t^{*}\right)$. Indeed, if the preceding property holds, the function $F^{*}(t)$ taking the values $\beta$ at $t=t^{*}$ and $F(t)$ elsewhere is obviously a discontinuous solution of (13), and the converse is clear. It can be seen, for example, numerically, that $A_{t}$ contains two elements for $t$ large enough.

2.2. The hyperbolic muscle. The presence of $F(t)$ in the kernel of (13) rules out most of the arguments of the classical theory of integral equations. It is not clear whether this equation admits a solution or not, and this point is important because, in our model, (13) governs the activation of a muscle.

Since $\rho$ is strictly positive a.e., the function $H(t)=\int_{t_{\min }}^{t} h(s) d s, t \in\left[t_{\min }, t_{\text {max }}\right]$, is strictly increasing and hence invertible. Let us note that $H(t)$ is the force of the muscle when all MUs up to level $t$ produce their tetanic force and $H\left(t_{\max }\right)=F_{\max }$.

By introducing $K(a, b)=1-c \exp \left(-\alpha \frac{b-a}{a+\Delta}\right), a, b \in \mathbb{R}_{+}=[0,+\infty)$, (13) can be written $F(v)=\int_{t_{\text {min }}}^{v} K(F(u), F(v)) h(u) d u$. Since $H$ is strictly increasing and absolutely continuous, the change of variable $u=H^{-1}(s)$ leads to $F(v)=\int_{H\left(t_{\text {min }}\right)}^{H(v)}$ $K\left(F\left(H^{-1}(s)\right), F(v)\right) d s$ for $v \in\left[t_{\min }, t_{\max }\right]$. Without risk of confusion we write $H(v)=t$ and because $H\left(t_{\min }\right)=0, H\left(t_{\max }\right)=F_{\max }$, we obtain $F\left(H^{-1}(t)\right)=$ $\int_{0}^{t} K\left(F\left(H^{-1}(s)\right), F\left(H^{-1}(t)\right)\right) d s$ for $t \in\left[0, F_{\max }\right]$. By defining $Y(t):=F\left(H^{-1}(t)\right)$ and $T=F_{\max }$, we get

$$
Y(t)=\int_{0}^{t}\left(1-c \exp \left(-\alpha \frac{Y(t)-Y(s)}{Y(s)+\Delta}\right)\right) d s, \quad t \in[0, T] .
$$

Since (14) describes the activation of a muscle whose MU density is the hyperbola $\rho(t)=\frac{1}{t}(t>0)$, it is natural to call it hyperbolic. Clearly, such a muscle has no physiological reality since any interval $(0, T]$ contains infinitely many of its MUs. This theoretical muscle is nevertheless interesting. By playing with the notation, we get

$$
F(t)=Y(H(t)), \quad t \in\left[t_{\min }, t_{\max }\right]
$$

and we see that the force of an arbitrary muscle can be deduced from that of a hyperbolic one. It is therefore enough to study (14).

2.3. Existence and unicity of a physiological solution. Straightforward computations provide the following theorem.

Theorem 1. Let $0<c<1, \alpha>0, \Delta>0$, and $a, b \in \mathbb{R}_{+} \mapsto K(a, b)=$ $1-c \exp \left(-\alpha \frac{b-a}{a+\Delta}\right)$.

(a) $K(a, b)$ together with the partial derivatives $K_{a}(a, b), K_{b}(a, b)$, and $K_{b b}(a, b)$ are continuous and bounded.

(b) $K_{a}(a, b)<0, K_{b}(a, b)>0, K_{b b}(a, b)<0$, and $K(a, b)$ is thus a concave function in the variable $b$.

We first prove the existence and unicity of a nonnegative solution of (14) for small values of $T$. For $0<T<\infty$, the set $E_{T}=\{f:[0, T] \mapsto \mathbb{R} ; f$ is measurable and bounded\}, equipped with the metric $d(f, g):=\sup _{t \in[0, T]}|f(t)-g(t)|$, is a complete metric space. Since uniform convergence preserves nonnegativity, $E_{T}^{+}=\left\{f \in E_{T} ; f \geq\right.$ $0\}$ is a closed subset of $E_{T}$ and hence also a complete metric space. Clearly, a 
solution of (14) is a fixed point of the operator $A_{T} f(t)=\int_{0}^{t} K(f(s), f(t)) d s, t \in$ $[0, T], f \in E_{T}^{+}$, which maps $E_{T}^{+}$into itself. According to Theorem 1, the mean value theorem can be applied to $K(a, b)$ and provides, for any $f, g \in E_{T}^{+}: d\left(A_{T} f, A_{T} g\right) \leq$ $2 T M d(f, g)$, where $M:=\sup _{a, b \in \mathbb{R}_{+}}\left(\left|K_{a}(a, b)\right|,\left|K_{b}(a, b)\right|\right)$. We conclude that $A_{T}$ admits a unique fixed point for $T<\frac{1}{2 M}$ since it is a contraction, and we prove the following theorem.

ThEOREM 2. For $T<\frac{1}{2 M}$, (14) admits a unique nonnegative solution in $E_{T}^{+}$.

Physiologically, a solution $Y(t)$ of (14) represents the force developed by a hyperbolic muscle, all of whose MUs, up to level $t$, are recruited. Therefore, $Y$ has to be a nondecreasing function with $Y(0)=0$, and any such solution will be called physiological. Since $0<1-c \leq K(a, b) \leq 1$ for $0 \leq a \leq b$, we get $0 \leq Y(t) \leq t$ for $t \in[0, T]$, and $Y$ is an element of $S_{T}:=\left\{f \in E_{T} ; f\right.$ is nondecreasing, $0 \leq f(t) \leq t$, $t \in[0, T]\}$. Clearly, $S_{T} \subset E_{T}^{+}$and every element $f$ of $S_{T}$ satisfies $f(0)=0$.

We now prove the existence of a physiological solution of (14) for an arbitrary $T>0$ and start by recalling the following.

TheOrem of Schauder (see [41]). Any nonempty compact and convex subset of a normed space has the fixed point property; i.e., every continuous mapping of such a subset into itself has at least one fixed point.

TheOREM of Helly (see [6]). Let $\left(f_{n}\right)_{n \in \mathbb{N}}$ be a sequence of uniformly bounded, nondecreasing, and right continuous functions defined over an interval in $\mathbb{R}$. Then there exist a subsequence $\left(f_{n_{k}}\right)_{k \in \mathbb{N}}$ and a nondecreasing right continuous function $f$ such that $\lim _{k \rightarrow \infty} f_{n_{k}}(t)=f(t)$, for all continuity points $t$ of $f$.

THEOREM 3. Every sequence in $S_{T}$ contains a subsequence converging in the mean of order 1 to an element of $S_{T}$. Furthermore, $S_{T}$ is nonempty and convex.

Proof. For any sequence $\left(f_{n}\right)_{n \in \mathbb{N}}$ in $S_{T}$, the functions $t \in[0, T] \longmapsto f_{n}^{+}(t)=$ $\lim _{h \downarrow 0} f_{n}(t+h)$ are also in $S_{T}$ and right continuous. According to Helly's theorem, there exist a subsequence $\left(n_{k}\right)_{k \in \mathbb{N}}$ and a nondecreasing right continuous function $f^{+}$ such that $f_{n_{k}}(t)$ converges to $f^{+}(t)$, as $k \rightarrow \infty$, for every continuity point $t$ of $f^{+}$in $[0, T]$. Since $f^{+}$is in $S_{T}$ and the exceptional set is at most countable, the convergence takes place a.e. and hence in measure. Furthermore, the sequence, being uniformly bounded by $T$, is uniformly integrable, and we conclude to its convergence in the mean of order 1 . Because nonemptyness and convexity of $S_{T}$ are obvious, the proof is complete.

Since Schauder's theorem requires a normed space, we introduce $E_{T}^{*}=E_{T}(\bmod$ a.e.) equipped with the $L_{1}$ norm. Let us recall that an element of $E_{T}^{*}$ is an equivalence class of functions which are equal a.e. to a representative. Hence, $S_{T}^{*}$ is the subset of $E_{T}^{*}$ such that each equivalence class contains an element of $S_{T}$. $S_{T}^{*}$ is nonempty and convex. Since every sequence in $S_{T}^{*}$ has a corresponding sequence of representatives in $S_{T}, S_{T}^{*}$ is also a compact subset of the normed space $E_{T}^{*}$ as a consequence of the first part of Theorem 3 .

Let $f^{*}$ be the equivalence class in $S_{T}^{*}$ of an element $f \in S_{T}$. Since $f=f^{\prime}$ (a.e.) for elements in $S_{T}$ implies that $A_{T} f=A_{T} f^{\prime}$ (a.e.), we can define $A_{T}^{*}$ over $S_{T}^{*}$ with $A_{T}^{*} f^{*}=\left(A_{T} f\right)^{*}$ because it is independent of the representative.

THEOREM 4. $A_{T}^{*}$ is a continuous mapping of $S_{T}^{*}$ into itself.

Proof. Let $f$ be a nondecreasing representative of $f^{*}$ in $S_{T}$. Since $0<1-c \leq$ $K(a, b) \leq 1$ for $0 \leq a \leq b$, we get $0 \leq A_{T} f(t) \leq t$ for $t \in[0, T]$. Furthermore, $K(a, b)$ is nonnegative and increasing in the second variable for $a, b \geq 0$, and consequently, for $0 \leq t \leq t^{\prime} \leq T$, we have $A_{T} f(t)=\int_{0}^{t} K(f(s), f(t)) d s \leq \int_{0}^{t^{\prime}} K\left(f(s), f\left(t^{\prime}\right)\right) d s=$ 
$A_{T} f\left(t^{\prime}\right)$. Therefore, $A_{T} f$ is an element of $S_{T}$ and $A_{T}^{*} f^{*}$ belongs to $S_{T}^{*}$ since a.e. equalities are preserved by $A_{T}$. To prove the continuity assertion, let us consider a sequence $\left(f_{n}^{*}\right)_{n \in \mathbb{N}}$ in $S_{T}^{*}$ which converges to $\tilde{f}^{*}$ in $L_{1}$. Since $S_{T}^{*}$ is closed as a compact subset of a Hausdorff space, we conclude that $\tilde{f}^{*}$ belongs to $S_{T}^{*}$. Furthermore, convergence in $L_{1}$ entails convergence in measure, and, for any $\sigma$-finite measure, the latter is equivalent to the statement "every subsequence contains a subsequence converging a.e." By using this property and the boundedness and continuity of $K(.,$.$) , we get the desired$ result via the dominated convergence theorem.

TheOrem 5. For every $T>0$, (14) admits a physiological solution.

Proof. Schauder's theorem provides the existence of a fixed point $f^{*}$ of $A_{T}^{*}$ in $S_{T}^{*}$. For any nondecreasing representative $f$ of the latter, we have $A_{T} f=f$ (a.e.) in $[0, T]$. The exceptional set $E$ having measure 0 , its complementary $E^{c}$ is dense in $[0, T]$, and for every $t \in E^{c}$, one can define $f_{-}(t)$ as the left-hand limit of $f$ over $E^{c}$ since $f$ is nondecreasing and bounded. The function $\tilde{f}(t):=\lim _{s \uparrow t} f(s)$ is nonnegative and nondecreasing. By using the boundedness and continuity of $K(.,$.$) and the dominated$ convergence theorem, one can show that $\tilde{f}$ satisfies $A_{T} \tilde{f}(t)=\tilde{f}(t)$ for every $t$ in $[0, T]$, and the proof is complete.

We prove that increasingness and continuity are equivalent properties for solutions of (14).

Lemma 6. Let $f:[a, b] \mapsto \mathbb{R}$ be continuous and $f(a)=f(b)$. For every $\delta>0$, there exist $a \leq t<t^{\prime} \leq b$ with $0<t^{\prime}-t<\delta$ and $f(t)=f\left(t^{\prime}\right)$.

Proof. The property clearly holds if $f$ is constant. Otherwise, replacing $f$ by $-f$ if necessary, we can assume that $f$ admits a maximum $M \neq f(a)$ at $t^{*}$ in the open interval $(a, b)$. Choosing $\lambda<M$ sufficiently close to $M$, the intermediate value theorem for continuous functions implies the existence of $a<t<t^{*}<t^{\prime}<b$, such that $f(t)=f\left(t^{\prime}\right)=\lambda$ and $t, t^{\prime}$ are as close as we want to $t^{*}$. Let us note that if $f$ is not constant, it is even possible to choose $t$ and $t^{\prime}$ so that $f(s) \neq f(a)$ for all $s \in\left[t, t^{\prime}\right]$.

THEOREM 7. A continuous solution of (14) starting at 0 is strictly increasing.

Proof. We first show that strict positivity of a continuous solution $Y$ of (14) over an interval $J \subset(0, T]$ entails its injectivity. Assuming the contrary, one can find $a<b$ in $J$ with $Y(a)=Y(b)$. According to the preceding lemma, there exist $t<t^{\prime}$ in $J$, as close as we want, with $Y(t)=Y\left(t^{\prime}\right)=\lambda>0$ and, from (14), we deduce $0=Y\left(t^{\prime}\right)-Y(t)=\int_{t}^{t^{\prime}} K(Y(s), \lambda) d s$. Since $K(\lambda, \lambda)>0$ and $K$ is continuous over $\mathbb{R}_{+}^{2}, K(Y(s), \lambda)>0$ over $\left[t, t^{\prime}\right]$ for $t, t^{\prime}$ sufficiently close to each other. Therefore, the preceding integral is positive and we get a contradiction.

To prove the theorem, we observe that the form of the kernel $K$ implies the existence of a neighborhood of $(0,0)$ in $\mathbb{R}^{2}$ over which $K(.,)>$.0 . Since $Y(0)=0$ and $Y$ is continuous, there exists $0<t_{0}<T$, such that for all $0 \leq s \leq t \leq t_{0}$, $K(Y(s), Y(t))>0$ and hence $Y(t)>0$ over $\left(0, t_{0}\right]$. Consequently, $Y$ is injective and hence increasing over $\left[0, t_{0}\right]$. A first zero of $Y$ after $t_{0}$ would violate its injectivity over an interval of positivity, and the proof is complete.

The following lemma will be used to discuss the converse of the preceding result. In what follows, we shall use the notation $f_{x}:=\frac{\partial f}{\partial x}$ for a function $f$.

LEMma 8. Let $Y, Y(0)=0$, be a nondecreasing solution of (14) defined over some interval $[0, T]$ and $G(a, b):=\int_{0}^{a} K(Y(s), b) d s$ for $(a, b) \in[0, T] \times \mathbb{R}$. The function $G$ has the following properties: (a) For every fixed $a \in[0, T], G(a, b)$ is a strictly increasing and concave function of $b$. (b) $G(a, b)$ is continuous. (c) $G_{b}(a, b)$ 
is continuous, and the same property holds for $G_{a}(a, b)$ if $Y$ is continuous. (d) If $Y$ is continuous, so are $G(t, Y(t))$ and $G_{b}(t, Y(t))$ as functions of $t$.

Proof. Strict monotonicity in (a) is obvious and concavity follows from the fact that it is preserved by integration. Properties (b) and (c) can be deduced from the dominated convergence theorem and suitable estimations, and (d) is obvious.

ThEOREM 9. Any nondecreasing solution of (14) starting at 0 is continuously differentiable.

Proof. Let $\tilde{Y}$ defined over $[0, T](\tilde{Y}(0)=0)$ be a nondecreasing solution of (14) and $Y$ the function of its right-hand limits. $Y$ is nondecreasing and right continuous, and, by a density argument, continuity of $\tilde{Y}$ and $Y$ are equivalent. Moreover, the dominated convergence theorem provides $Y(t)=\int_{0}^{t} K(\tilde{Y}(s), Y(t)) d s$ and since $\tilde{Y}(t)=$ $Y(t)$ (a.e.), $Y(t)$ is also a solution of (14). Because of the concavity property (a) in Lemma 8 , for every $t \in[0, T], G(t, y)=y$ admits at most two solutions, one of them being $Y(t)$ since $G(t, Y(t))=Y(t)$. Moreover, if for a given $t$ the preceding equation has two distinct solutions $y_{1}(t)<y_{2}(t)$, then $G_{b}\left(t, y_{2}(t)\right)<1$ and $G_{b}\left(t, y_{1}(t)\right)>1$.

According to Theorem 2, over a sufficiently small right-hand interval of $0,(14)$ admits a unique nonnegative solution in the space of bounded measurable functions. As a consequence, $Y(t)$ is the unique solution of $G(t, y)=y$ for $t$ small enough. The function $G$ satisfies all regularity conditions of the implicit function theorem [41]. Since $G(0,0)=0$ and $G_{b}(0,0)=0<1$, the latter entails the existence of a unique continuous function $y$ such that $y(0)=0$ and $G(t, y(t))-y(t)=0$ over a right-hand neighborhood of 0 . The preceding unicity property implies $Y \equiv y$ for $t$ small enough and continuity entails $G_{b}(t, Y(t))<1$ over some right-hand interval of 0 . Hence there is an interval $[0, \beta)$ of maximal length $\beta$ over which $G_{b}(t, Y(t))<1$.

If $Y$ is not continuous over $[0, \beta)$, then there is a point of discontinuity $t_{0} \neq 0$ because $Y$ is continuous for small values of $t$. $Y$ is right continuous and since it is increasing, its left and right-hand limits at $t_{0}$ satisfy $Y\left(t_{0}^{-}\right)<Y\left(t_{0}^{+}\right)=Y\left(t_{0}\right)$. According to the implicit function theorem, there exists a unique continuous function $y_{0}$ defined over an open interval $I_{t_{0}}$ containing $t_{0}$, such that $y_{0}\left(t_{0}\right)=Y\left(t_{0}\right)$ and $G\left(t, y_{0}(t)\right)=y_{0}(t)$ for $t \in I_{t_{0}}$. Since $G_{b}\left(t_{0}, Y\left(t_{0}\right)\right)<1$, the continuity of $y_{0}$ implies the existence of $t<t_{0}$ in $I_{t_{0}}$, with $G_{b}\left(t, y_{0}(t)\right)<1$ and $Y\left(t_{0}^{-}\right)<y_{0}(t)$. Because $Y(t) \leq Y\left(t_{0}^{-}\right)$, we obtain the configuration $G(t, Y(t))=Y(t), G\left(t, y_{0}(t)\right)=y_{0}(t), G_{b}(t, Y(t))<1, G_{b}\left(t, y_{0}(t)\right)<1$, and $Y(t) \neq y_{0}(t)$. This is a contradiction of the concavity of $G(t, b)$ in the variable $b$, and $Y$ is continuous over $[0, \beta)$ and thus identical to $\tilde{Y}$.

The function $G_{b}(a, b)$ is continuous and, because of (c) in Lemma 8, the continuity of $Y$ implies that of $G_{a}(a, b)$. Consequently, $G$ is continuously differentiable and, according to another version of the implicit function theorem [10, 11], every locally defined continuous solution $y$ provided by the last theorem is also differentiable. Since $Y$ is continuous over $[0, \beta)$, local unicity implies local identity of $y$ and $Y$ and entails the differentiability of the latter over $[0, \beta)$.

In the following considerations, every interchange of integration and derivation can be justified with the dominated convergence theorem. For $t \in[0, T]$ we introduce $\phi(t):=G_{b}(t, Y(t))=\int_{0}^{t} \frac{\alpha c}{Y(s)+\Delta}\left(\exp \left(-\alpha \frac{Y(t)-Y(s)}{Y(s)+\Delta}\right)\right) d s$. Writing ' $=\frac{d}{d t}$ and using the differentiability of $Y$, for every $t \in[0, \beta)$ we get

$$
\phi^{\prime}(t)=K_{b}(Y(t), Y(t))+\frac{1-c}{1-\phi(t)} \int_{0}^{t} K_{b b}(Y(s), Y(t)) d s .
$$

We now suppose that $\beta<T$ and claim that $\sup _{t \in[0, \beta)} \phi(t)=m<1$. If not, $m=1$ as a consequence of the definition of $\beta$, and by using the mean value theorem 
of differential calculus, one can choose an increasing sequence $\left(t_{n}\right)_{n \in \mathbb{N}}$ such that $t_{n} \uparrow \beta$ as $n \rightarrow \infty$ and $\phi^{\prime}\left(t_{n}\right) \geq 0$ for every $n \in \mathbb{N}$. The first term in (16) is positive and bounded, and the integral is negative and bounded away from 0 . Since $0<c<1$, the quotient before the integral tends to $+\infty$ as $t \uparrow \beta$. According to (16), $\phi^{\prime}\left(t_{n}\right)<0$ for $n$ sufficiently large, and we get a contradiction and conclude that $m<1$.

Because of the monotonicity of $Y, Y(t) \leq Y(\beta)$ for all $t \in[0, \beta)$, and thus, for any sequence $u_{n} \uparrow \beta$, we have $\int_{0}^{u_{n}} \frac{\alpha c}{Y(s)+\Delta}\left(\exp \left(-\alpha \frac{Y(\beta)-Y(s)}{Y(s)+\Delta}\right)\right) d s \leq \phi\left(u_{n}\right) \leq m$. Letting $n \rightarrow \infty$ in the integral, we get $\phi(\beta) \leq m<1$ and thus $G_{b}(\beta, Y(\beta))<1$. Applying once more the implicit function theorem, we get a unique continuous function $y^{+}$ defined over an open interval $I_{\beta}$ containing $\beta$ with $y^{+}(\beta)=Y(\beta), G\left(t, y^{+}(t)\right)=y^{+}(t)$ over $I_{\beta}$, and $G_{b}\left(t, y^{+}(t)\right)<1$ for every $t$ sufficiently close to $\beta$. If $Y \equiv y^{+}$does not hold over a right-hand open neighborhood of $\beta$, then one can find a sequence $v_{n} \downarrow \beta$ as $n \rightarrow \infty$, such that $Y\left(v_{n}\right) \neq y^{+}\left(v_{n}\right)$ for every $n$. Monotonicity and concavity entail $Y(\beta) \leq Y\left(v_{n}\right)<y^{+}\left(v_{n}\right)$, and hence $Y\left(v_{n}\right) \rightarrow Y(\beta)$ as $n \rightarrow \infty$. Concavity again implies that $G_{b}\left(v_{n}, Y\left(v_{n}\right)\right)>1$, and thus, letting $n \rightarrow \infty, G_{b}(\beta, Y(\beta)) \geq 1$, which is a contradiction. Since $Y \equiv y^{+}$on a right-hand open interval of $\beta, G_{b}(t, Y(t))<1$ for all sufficiently small $t$ to the right of $\beta$, which is a contradiction of its maximality. Consequently $\beta=T$ and $Y$ is continuous over $[0, T]$. Moreover, $Y$ is also differentiable, and the relations $Y^{\prime}(t)=\frac{1-c}{1-\phi(t)}=\frac{1-c}{1-G_{b}(t, Y(t))}$ show that it is even continuously differentiable and $Y^{\prime}(t)>0$ over $[0, T]$.

We would like to stress that, in Theorem 9, the condition $0<c<1$ plays a delicate role. Indeed, let us assume that $c=1$ and look for a solution of (14) of the form $Y(t)=a I_{\left(t^{*},+\infty\right)}(t)$, where $I$ denotes the indicator function and $t^{*}$ and $a$ have to be specified. For $t \leq t^{*},(14)$ is satisfied since both sides are equal to 0 . For $t>t^{*}$, it reduces to $a=t^{*}\left(1-\exp \left(-\frac{\alpha a}{\Delta}\right)\right)$. By choosing $t^{*}$ so that $\frac{t^{*} \alpha}{\Delta}>1$ and the positive solution of the preceding equation for $a$, we get a discontinuous nondecreasing solution of (14).

Collecting all the preceding results, we get the following theorem.

THEOREM 10. The following properties are equivalent for a solution $Y$ of (14) with $Y(0)=0$ : (a) $Y$ is physiological, i.e., nondecreasing, (b) $Y$ is strictly increasing, (c) $Y$ is continuous, and (d) $Y$ is continuously differentiable and $Y^{\prime}$ is strictly positive.

It is interesting to note that although (14) admits possibly discontinuous solutions, any physiological solution is automatically continuously differentiable.

THEOREM 11. Equation (14) admits a unique physiological solution.

This uniqueness result can be deduced from Gronwall's inequality and the fact that, according to the proof of Theorem $9, \phi(t)<1$ over $[0, T]$. We propose another argument which will also provide a nice way to get the solution of (14).

Proof. We proved that every nondecreasing solution $Y$ of (14) over $[0, T]$ is strictly increasing and continuously differentiable with a strictly positive and bounded derivative. Consequently, $X=Y^{-1}$ has the same properties as $Y$ and $Y(X(t))=t$ for every $t \geq 0$. In particular, the derivative of $X$, denoted $x$, is continuous. Performing the change of variables $s=X(u)$ and $v=X(t)$ in (14) and rewriting $s$ and $t$ instead of $u$ and $v$, we get

$$
t=\int_{0}^{t} x(s)\left(1-c \exp \left(-\alpha \frac{t-s}{s+\Delta}\right)\right) d s
$$

Equation (17) is a linear Volterra equation of the first kind for $x$, the derivative of $X$ (inverse function of $Y$ ). Since the kernel of (17) satisfies all the conditions of Theorem 
1.3.5 in [4], this equation admits a unique continuous solution, and thus Theorem 11 is proved.

It is interesting to note that (17) has an interpretation. Indeed, it is an integral equation for the force density function $x$ of a muscle whose force is $t$ if its MUs up to level $t$ are recruited.

For continuous $x$, derivation and integration by parts of (17) provide, respectively,

$$
\begin{aligned}
& x(t)=\frac{1}{1-c}-\frac{\alpha c}{1-c} \int_{0}^{t} x(s) \frac{1}{s+\Delta} \exp \left(-\alpha \frac{t-s}{s+\Delta}\right) d s \text { and } \\
& X(t)=\frac{t}{1-c}-\frac{\alpha c}{1-c} \int_{0}^{t} X(s) \frac{t+\Delta}{(s+\Delta)^{2}} \exp \left(-\alpha \frac{t-s}{s+\Delta}\right) d s .
\end{aligned}
$$

The last two equations are linear Volterra equations of the second kind, for which theory and numerical treatments are well known [4, 14, 32].

Let us remark that existence and unicity of $Y$ for every $T>0$ automatically provide a unique solution of (14) defined over $[0, \infty)$.

We would like to point out that Theorem 10 together with the preceding arguments also lead to the existence of an increasing and continuously differentiable solution of (14). However, Schauder's theorem provides the existence of a nondecreasing solution without continuity assumption and thus without Theorem 10. Both approaches are interesting, and methodological diversity is always welcome for the treatment of future investigations.

3. Properties of the physiological solution. In the following, $x$ will be the unique continuous solution of (17) defined over $[0,+\infty)$ and $X(t)=\int_{0}^{t} x(s) d s$. As can be seen in (18), $x$ depends on the parameters $\alpha, c$, and $\Delta=k I n_{0}$. Since $\alpha$ and $c$ are fixed and muscle-independent, $x, X$, and $Y$ will be considered as functions of the two variables $t \geq 0$ and $\Delta>0$ and written $x(t, \Delta), X(t, \Delta)$, and $Y(t, \Delta)$.

THEOREM 12. For every fixed $\Delta>0$, the following properties hold: (a) $x(t, \Delta)$ is continuously differentiable in the variable $t$, (b) $x(0, \Delta)=\frac{1}{1-c}$, (c) $0<x(t, \Delta) \leq$ $(1-c)^{-1}$ for every $t>0$, (d) $0<X(t, \Delta) \leq \frac{t}{1-c}$ for every $t>0$, (e) as a function of $t, \frac{X(t, \Delta)}{t}$ is decreasing over $(0, \infty)$, and (f) as a function of $u, \frac{Y(u, \Delta)}{u}$ is increasing over $(0, \infty)$.

Proof. Part (a) follows from the facts that (18) is again differentiable in $t$ and that the result involves only continuous functions. Since the integrand in (18) is bounded, letting $t \rightarrow 0$, we get (b). The second inequality in (c) follows from (18) and $x(t, \Delta)=X_{t}(t, \Delta)>0$. Integrating (c) provides (d). To prove (e), we have to show that $\frac{\partial}{\partial t}\left(\frac{X(t, \Delta)}{t}\right)=\frac{1}{t}\left(x(t, \Delta)-\frac{X(t, \Delta)}{t}\right) \leq 0$, that is, $\frac{X(t, \Delta)}{t} \geq x(t, \Delta)$ for $t>0$. We divide (19) by $t$ and obtain $\frac{X(t, \Delta)}{t}=\frac{1}{1-c}+\int_{0}^{t} K(s, t) \frac{s}{t} \frac{t+\Delta}{s+\Delta} \frac{X(s, \Delta)}{s} d s=$ $\frac{1}{1-c}+\int_{0}^{t} \tilde{K}(s, t) \frac{X(s, \Delta)}{s} d s$, where $K(s, t):=-\frac{\alpha c}{1-c} \frac{1}{s+\Delta} \exp \left(-\alpha \frac{t-s}{s+\Delta}\right)$ is the kernel of (18). We observe that $\tilde{K}(s, t)=K(s, t) \frac{s}{t} \frac{t+\Delta}{s+\Delta}$. A straightforward computation based on kernel iterations shows that the corresponding resolvents $R(s, t)$ and $R^{\prime}(s, t)$ also satisfy $R^{\prime}(s, t)=R(s, t) \frac{s}{t} \frac{t+\Delta}{s+\Delta}$. Obviously for $\Delta>0$ and $0<s \leq t$, we have $\frac{s}{t} \frac{t+\Delta}{s+\Delta}<1$, and according to the general theory [4], we can write $\frac{X(t, \Delta)}{t}=$ $(1-c)^{-1}+(1-c)^{-1} \int_{0}^{t} R^{\prime}(s, t) d s=(1-c)^{-1}+(1-c)^{-1} \int_{0}^{t} R(s, t) \frac{s}{t} \frac{t+\Delta}{s+\Delta} d s \geq(1-c)^{-1}+$ $(1-c)^{-1} \int_{0}^{t} R(s, t) d s=x(t, \Delta)$. The last inequality clearly holds if $R(s, t) \leq 0$, and it is enough to prove the latter. The resolvent formula [4] provides $-R(s, t)=$ $\frac{\alpha c}{1-c} \frac{1}{s+\Delta} \exp \left(-\alpha \frac{t-s}{s+\Delta}\right)+\int_{s}^{t} R(s, u) \frac{\alpha c}{1-c} \frac{1}{u+\Delta} \exp \left(-\alpha \frac{t-u}{u+\Delta}\right) d u$. Applying the changes of 
variables $u=v-\Delta$ and then $v=\exp (w)$ and rewriting $t^{\prime}=\ln (t+\Delta), s^{\prime}=\ln (s+\Delta)$, for fixed $s^{\prime},-R^{\prime}\left(s^{\prime}, t^{\prime}\right)=-R\left(\exp \left(s^{\prime}\right)-\Delta, \exp \left(t^{\prime}\right)-\Delta\right)$ is a solution of the convolutiontype integral equation $-R^{\prime}\left(s^{\prime}, t^{\prime}\right)=f\left(t^{\prime}\right)+\int_{s^{\prime}}^{t^{\prime}} R^{\prime}\left(s^{\prime}, w\right) h\left(t^{\prime}-w\right) d w$, where $f\left(t^{\prime}\right)=$ $\frac{\alpha c}{1-c} \frac{1}{\exp \left(s^{\prime}\right)} \exp \left(-\alpha \frac{\exp \left(t^{\prime}\right)-\exp \left(s^{\prime}\right)}{\exp \left(s^{\prime}\right)}\right)$ and $h\left(t^{\prime}\right)=\frac{\alpha c}{1-c} \exp \left(\alpha-\alpha \exp \left(t^{\prime}\right)\right)$. Since $\frac{f(T)}{f(t)}=$ $\exp \left(-\alpha e^{-s^{\prime}}\left(e^{T}-e^{t}\right)\right) \leq \exp \left(-\alpha e^{-s}\left(e^{T}-e^{t}\right)\right)=\frac{h(T-s)}{h(t-s)}$ for $0 \leq s^{\prime} \leq s<T<t$, according to Theorem 6.1 in [32], we conclude to $R^{\prime}\left(s^{\prime}, t^{\prime}\right) \leq 0$.

For $t_{1}=Y\left(u_{1}, \Delta\right)$ and $t_{2}=Y\left(u_{2}, \Delta\right), t_{1}<t_{2}$ implies $u_{1}<u_{2}$. By using (e) and $Y(t, \Delta)=X^{-1}(t, \Delta)$ in the variable $t$, we get $\frac{u_{1}}{Y\left(u_{1}, \Delta\right)}=\frac{X\left(Y\left(u_{1}, \Delta\right), \Delta\right)}{Y\left(u_{1}, \Delta\right)}=\frac{X\left(t_{1}, \Delta\right)}{t_{1}} \geq$ $\frac{X\left(t_{2}, \Delta\right)}{t_{2}}=\frac{X\left(Y\left(u_{2}, \Delta\right), \Delta\right)}{Y\left(u_{2}, \Delta\right)}=\frac{u_{2}}{Y\left(u_{2}, \Delta\right)}$, and (f) is proved.

TheOREm 13. For every $\lambda>0, t \geq 0, u \geq 0$, and $\Delta>0$, we have (a) $x(t, \lambda \Delta)=$ $x\left(\frac{t}{\lambda}, \Delta\right)$, (b) $x(\lambda t, \Delta)=x\left(t, \frac{\Delta}{\lambda}\right)$, (c) $x(\lambda t, \lambda \Delta)=x(t, \Delta)$, (d) $X(\lambda t, \lambda \Delta)=\lambda X(t, \Delta)$, (e) $Y(\lambda u, \lambda \Delta)=\lambda Y(u, \Delta)$, and (f) $Y_{\Delta}(u, \Delta)+u Y_{u}(u, \Delta)=Y(u, \Delta)$.

Proof. For every $\lambda>0,(17)$ yields $t=\int_{0}^{t} x(s, \Delta)\left(1-c \exp \left(-\alpha \frac{\lambda t-\lambda s}{\lambda s+\lambda \Delta}\right)\right) d s$. By using the change of variable $s=\frac{z}{\lambda}$ and ultimately replacing $\lambda t$ by $t$, we get $t=$ $\int_{0}^{t} x\left(\frac{z}{\lambda}, \Delta\right)\left(1-c \exp \left(-\alpha \frac{t-z}{z+\lambda \Delta}\right)\right) d z$. However, according to (17), we also have $t=$ $\int_{0}^{t} x(z, \lambda \Delta)\left(1-c \exp \left(-\alpha \frac{t-z}{z+\lambda \Delta}\right)\right) d z$. Since (17) admits a unique continuous solution, we conclude that $x(z, \lambda \Delta)=x\left(\frac{z}{\lambda}, \Delta\right)$ for all possible values of their arguments. This is equivalent to (a) and (b), and writing $z=\lambda s$ yields (c). Part (d) follows from $X(\lambda t, \lambda \Delta)=\int_{0}^{\lambda t} x\left(\frac{z}{\lambda}, \Delta\right) d z=\int_{0}^{\lambda t} x(z, \lambda \Delta) d z=\lambda \int_{0}^{t} x(s, \Delta) d s=\lambda X(t, \Delta)$. By using Theorem 13(d) and the fact that, for fixed $\Delta, Y$ is the inverse function of $X$, we can write $\lambda t=Y(X(\lambda t, \lambda \Delta), \lambda \Delta)=Y(\lambda X(t, \Delta), \lambda \Delta)=\lambda Y(X(t, \Delta), \Delta)$. The equality $Y(\lambda X(t, \Delta), \lambda \Delta)=\lambda Y(X(t, \Delta), \Delta)$ is valid for every $t \geq 0$. Because $X(t, \Delta)$ is strictly increasing in $t$, we substitute $u=X(t, \Delta)$, and (e) is proved. According to Theorem 13(e), $Y(u, \Delta)=\Delta Y\left(\frac{u}{\Delta}, 1\right)$, and the differentiability of $Y$ with respect to $u$ entails that with respect to $\Delta$.

For any differentiable function $F(u),\left.\frac{d}{d v} F(v)\right|_{\lambda u}=\frac{1}{\lambda} \frac{d}{d u} F(\lambda u)$, and thus $\left.Y_{v}(v, 1)\right|_{\frac{u}{\Delta}}$ $=\Delta Y_{u}\left(\frac{u}{\Delta}, 1\right)=Y_{u}(u, \Delta)$. By using Theorem 13(e) again, $Y_{\Delta}(u, \Delta)=\frac{\partial}{\partial \Delta}\left(\Delta Y\left(\frac{u}{\Delta}, 1\right)\right)=$ $Y\left(\frac{u}{\Delta}, 1\right)+\left.\Delta\left(-\frac{u}{\Delta^{2}}\right) Y_{v}(v, 1)\right|_{\frac{u}{\Delta}}$ and $Y_{\Delta}(u, \Delta)=\frac{1}{\Delta} Y(u, \Delta)-\frac{u}{\Delta} Y_{u}(u, \Delta)$. The last equality is equivalent to (f). The latter, which is the differential equivalent of (e), provides a partial differential equation for $Y$. Unfortunately, the initial condition is given along a characteristic curve and is equivalent to a solution of (17).

TheOREM 14. Let $x_{\infty}:=\left(1-c \int_{0}^{1} \exp \left(-\alpha \frac{1-s}{s}\right) d s\right)^{-1}$.

(a) For every $t \geq 0, \lim _{\Delta \uparrow \infty} x(t, \Delta)=\frac{1}{1-c}$.

(b) For every $t>0, \lim _{\Delta \downarrow 0} x(t, \Delta)=x_{\infty}$ and $\lim _{\Delta \downarrow 0} x(0, \Delta)=\frac{1}{1-c}$.

(c) For every $\Delta>0, \lim _{t \uparrow \infty} x(t, \Delta)=x_{\infty}$.

Proof. By using the continuity of $x, x(0, \Delta)=\frac{1}{1-c}$, and Theorem 13(a), we get $\frac{1}{1-c}=\lim _{t \downarrow 0} x(t, \Delta)=\lim _{\lambda \uparrow \infty} x\left(\frac{t}{\lambda}, \Delta\right)=\lim _{\lambda \uparrow \infty} x(t, \lambda \Delta)=\lim _{\Delta \uparrow \infty} x(t, \Delta)$, and (a) is proved.

It is enough to consider (b) for $t>0$. For every $0<t<t^{*}$ and $\Delta>0$, according to Theorem 13(c), we have $x(t, \Delta)=x\left(\frac{t}{t^{*}} t^{*}, \frac{t}{t^{*}} \frac{t^{*}}{t} \Delta\right)=x\left(t^{*}, \frac{t^{*}}{t} \Delta\right)$. Let $\Delta_{n} \downarrow 0$ and $x\left(t, \Delta_{n}\right) \rightarrow \liminf _{\Delta \downarrow 0} x(t, \Delta)$ as $n \uparrow \infty$. The preceding equality entails $\liminf \operatorname{ind}_{\Delta} x(t, \Delta)=\lim _{n \uparrow \infty} x\left(t, \Delta_{n}\right)=\lim _{n \uparrow \infty} x\left(t^{*}, \frac{t^{*}}{t} \Delta_{n}\right) \geq \liminf _{\Delta \downarrow 0} x\left(t^{*}, \Delta\right)$. Since for the same reasons the converse inequality also holds, we conclude that $\liminf _{\Delta \downarrow 0} x(t, \Delta)=\liminf _{\Delta \downarrow 0} x\left(t^{*}, \Delta\right)$. Hence, for $t>0, \liminf _{\Delta \downarrow 0} x(t, \Delta)$ is independent of $t$, and the same property holds for $\lim \sup _{\Delta \downarrow 0} x(t, \Delta)$. By using $\left(\Delta_{n}\right)$ such that $\lim _{n \rightarrow \infty} x\left(t, \Delta_{n}\right)=\underline{x},(17)$ and the dominated convergence theorem provide 

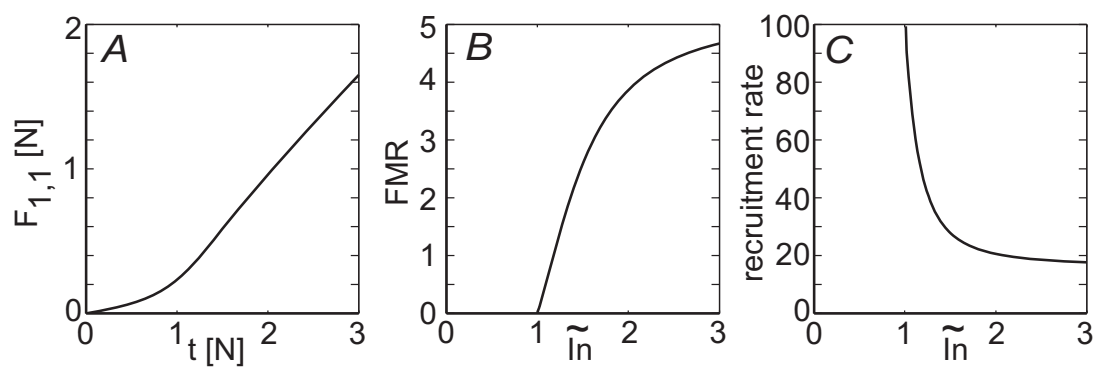

Fig. 2. A: Force of the standard hyperbolic muscle as a function of the tetanic force of the last recruited MU. B: Frequency modulation to recruitment ratio (FMR). C: Recruitment rate.

$t=\int_{0}^{t} \underline{x}\left(1-c \exp \left(-\alpha \frac{t-s}{s}\right)\right) d s$. Replacing $s$ by $s t$ in the preceding integral leads to $\underline{x}=\left(1-c \int_{0}^{1} \exp \left(-\alpha \frac{1-s}{s}\right) d s\right)^{-1}=x_{\infty}$. Since the same argument holds for $\bar{x}=\lim \sup _{\Delta \downarrow 0} x(t, \Delta)$, we conclude that $\underline{x}=\bar{x}=x_{\infty}$, and the proof of (b) is complete.

(c) follows from (b), with $\lim _{t \uparrow \infty} x(t, \Delta)=\lim _{\lambda \downarrow 0} x\left(\frac{t}{\lambda}, \Delta\right)=\lim _{\lambda \downarrow 0} x(t, \lambda \Delta)=$ $\lim _{\Delta \downarrow 0} x(t, \Delta)=x_{\infty}$.

The existence of the limits in the next theorem follows from the monotonicity properties discussed above.

TheOREM 15. For every $\Delta>0$, we have (a) $\lim _{t \uparrow \infty} \frac{X(t, \Delta)}{t}=x_{\infty}$, (b) $\lim _{t \downarrow 0} \frac{X(t, \Delta)}{t}$ $=\frac{1}{1-c}$, (c) $\lim _{u \uparrow \infty} \frac{Y(u, \Delta)}{u}=\frac{1}{x_{\infty}}$, and (d) $\lim _{u \downarrow 0} \frac{Y(u, \Delta)}{u}=1-c$.

Proof. (a) follows from Theorem 14(a) and the fact that ordinary convergence implies convergence in the Cesaro sense. Since $X(0, \Delta)=0$, the definition of the right-hand derivative at 0 provides (b). Part (d) follows from the same argument applied to the inverse function $Y$. To prove (c), for fixed $\Delta$ we substitute $t=Y(u, \Delta)$ in (a) and use $x_{\infty}=\lim _{t \uparrow \infty} \frac{X(t, \Delta)}{t}=\lim _{u \uparrow \infty} \frac{X(Y(u, \Delta), \Delta)}{Y(u, \Delta)}=\lim _{u \uparrow \infty} \frac{u}{Y(u, \Delta)}$.

3.1. Representation of the physiological solution. The use of Theorem 13 requires caution with units. For simplicity of notation, we introduce two rules:

- The argument of $F_{(h, \Delta)}$ and $\tilde{F}_{(h, \Delta)}$ is always expressed in Newtons.

- The presence or absence of units attributed to $F_{(h, \Delta)}$ and $Y$ is imposed by the context.

Since $\alpha$ and $c$ are muscle-independent, the solution $F$ of (13),

$$
F(t)=\int_{t_{\text {min }}}^{t} h(s)\left(1-c \exp \left(-\alpha \frac{F(t)-F(s)}{F(s)+\Delta}\right)\right) d s, \quad t \in\left[t_{\text {min }}, t_{\max }\right],
$$

depends only on $h$ and $\Delta$ and is therefore denoted $F_{(h, \Delta)}$. Recall that a muscle with $h \equiv 1$ was called hyperbolic, and with the new notation we have $F_{(1, \Delta)}(t)=Y(t, \Delta)$ for all $t \geq 0$. By using Theorem 13(e) and (15), we get $F_{(h, \Delta)}(t)=Y(H(t), \Delta)=$ $\Delta Y\left(\frac{H(t)}{\Delta}, 1\right)=\Delta F_{(1,1)}\left(\frac{H(t)}{\Delta}\right) . \quad F_{(1,1)}$ is the solution of $(13)$ for $h \equiv 1$ and $\Delta=1$, a muscle which will be called standard hyperbolic (Figure 2A). Introducing the relative force $\tilde{F}_{(h, \Delta)}(t):=\frac{F_{(h, \Delta)}(t)}{F_{\max }}$, we get

$$
\tilde{F}_{(h, \Delta)}(t)=\frac{\Delta}{F_{\max }} F_{(1,1)}\left(\frac{H(t)}{\Delta}\right)=\frac{\Delta}{F_{\max }} F_{(1,1)}\left(\frac{\frac{H(t)}{F_{\max }}}{\frac{\Delta}{F_{\max }}}\right)=A F_{(1,1)}\left(\frac{\tilde{H}(t)}{A}\right)
$$


where $\tilde{H}(t):=\frac{H(t)}{F_{\max }}$ and $A:=\frac{\Delta}{F_{\max }}$, a unit-free number. Thus

$$
\tilde{F}_{(h, \Delta)}(t)=A F_{(1,1)}\left(\frac{\tilde{H}(t)}{A}\right)
$$

shows that the solution of (13) for an arbitrary muscle can be derived from $F_{(1,1)}$, which is the inverse function of the solution $X(t, 1)$ of $(19)$ with $\Delta=1: X(t, 1)=\frac{t}{1-c}-$ $\frac{\alpha c}{1-c} \int_{0}^{t} X(s, 1) \frac{t+1}{(s+1)^{2}} \exp \left(-\alpha \frac{t-s}{s+1}\right) d s$. The values of $F_{(1,1)}$ can therefore be computed very accurately once and for all and then memorized for subsequent computations (Figure 2A).

The relative synaptic weight. By using $I n_{T}(t)=\frac{F_{(h, \Delta)}(t)}{k}+I n_{0},(7)$, and (20), the relative synaptic weight as a function of the tetanic force $t$ is given by

$$
\tilde{g}(t)=\frac{V_{T}}{\left(E_{E P S P}-V_{T}\right) \operatorname{In}_{0}\left(F_{(1,1)}\left(\frac{\tilde{H}(t)}{A}\right)+1\right)} .
$$

The range of $\tilde{g}$ is

$$
\left[\tilde{g}\left(t_{\text {max }}\right), \tilde{g}\left(t_{\text {min }}\right)\right]=\left[\frac{V_{T}}{\left(E_{E P S P}-V_{T}\right) \operatorname{In} n_{0}\left(F_{(1,1)}\left(\frac{1}{A}\right)+1\right)}, \frac{V_{T}}{\left(E_{E P S P}-V_{T}\right) I n_{0}}\right]
$$

and is thus independent of the shape of the MU distribution.

The trace of the muscle in $\tilde{g}(t)$ appears in $\tilde{H}(t)$ and $A$. According to the preceding results, there always exists a unique relative synaptic weight providing a given affine muscle response during recruitment. Since $F_{(1,1)}\left(\frac{\tilde{H}(t)}{A}\right)$ is strictly increasing in $t, \tilde{g}(t)$ is strictly decreasing. The integrability of $\rho$ and (21) imply that $\rho(t) \stackrel{\text { a.e. }}{=} \frac{\Delta}{t} \frac{d}{d t} F_{(1,1)}^{-1}\left(\frac{V_{T}}{\left(E_{E P S P}-V_{T}\right) I n_{0} \tilde{g}(t)}-1\right)$. For fixed values of $\Delta$ and $I n_{0}$ (or equivalently for fixed $k$ and $I n_{0}$ ) and once two densities equal a.e. have been identified, there is a one-to-one correspondence between the relative synaptic weight and the MU density function.

4. Activation of the muscle and related functionals. The study of the activation of a muscle is simplified by normalization of the input $\tilde{I} n=\frac{I n}{I n_{0}}$ and the muscle force $\tilde{\mathcal{F}}=\frac{\mathcal{F}}{F_{\max }}$. The curve given by $\tilde{I} n \mapsto \tilde{\mathcal{F}}(\tilde{I} n)$ will be called the relative activation curve. During recruitment, the latter is given by $\tilde{\mathcal{F}}(\tilde{I} n)=\frac{\Delta}{F_{\max }}(\tilde{I} n-1)$ (Figure 3A). The unit-free number $A=\frac{\Delta}{F_{\max }}$ in (20) is the slope of the preceding straight line and is called the activation factor (denoted $S$ in [42]). It is remarkable that $A$ depends on $\rho$ only through its first moment $F_{\text {max }}$.

Recruitment ratio. The recruitment ratio is the fraction $Q$ of the force at the end of recruitment and the maximal force of the muscle. As a ratio of two forces, $Q$ is a unit-free number. By using $(20)$, we can write $Q=A F_{(1,1)}\left(\frac{H\left(t_{\max }\right)}{\Delta}\right)$. Since $H\left(t_{\max }\right)=F_{\max }$ and $A=\frac{\Delta}{F_{\max }}$, the recruitment ratio (Figure $3 \mathrm{C}$ ) depends on $A$ only since

$$
Q(A)=A F_{(1,1)}\left(\frac{1}{A}\right) .
$$

Since $F_{(1, \Delta)}(t)=Y(t, \Delta), Q(A)=\frac{Y\left(\frac{1}{A}, 1\right)}{\frac{1}{A}}$, and because of Theorem $12(\mathrm{f})$, it is a decreasing function of $A$. Therefore, by using Theorem 15(c) and (d), for all $A>0$ 

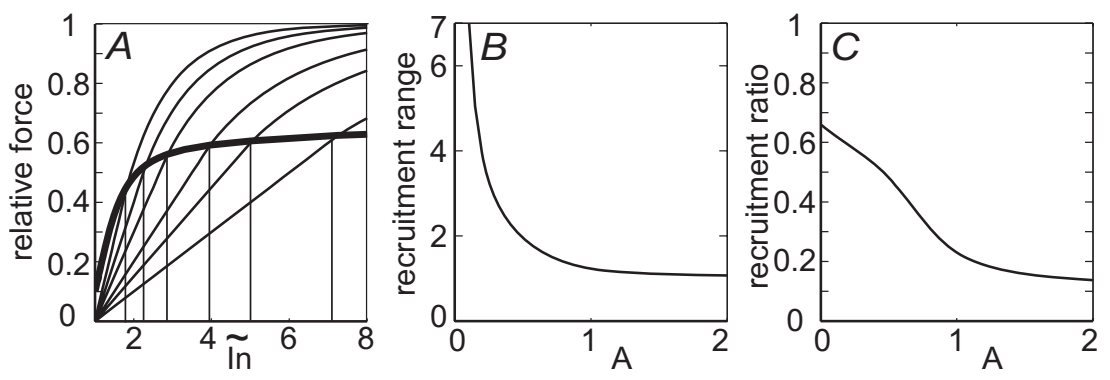

FIG. 3. A: Relative activation curves and end-recruitment curve as a function of the relative input. They are obtained by projection of the curves in Figure 4 in the (In, A) plane. B: Relative recruitment range $R(A)$. C: Recruitment ratio $Q(A)$.

we have $1-c=\lim _{u \downarrow 0} \frac{Y(u, 1)}{u} \leq Q(A) \leq \lim _{u \uparrow \infty} \frac{Y(u, 1)}{u}=x_{\infty}^{-1}$. For the estimated values $\alpha=1.14$ and $c=0.9$, we get $0.1 \leq Q(A) \leq 0.66$ for all $A>0$ and thus for all muscles.

Relative recruitment range. We call relative recruitment range $R$ the smallest relative input range within which all MUs are recruited. It is also the factor by which the threshold input of the smallest MU of the pool has to be multiplied in order to recruit all MUs. Since $\tilde{I} n_{T}\left(t_{\min }\right)=1$, we have $R=\tilde{I} n_{T}\left(t_{\max }\right)-1$. Since $A$ is the slope of the relative activation curve during recruitment (affine range), we have $R=\frac{Q}{A}$. Because of (22), $R$ is a function of $A$ only, given by $R=F_{(1,1)}\left(\frac{1}{A}\right)$ and thus decreasing (Figure 3B).

If $A \rightarrow \infty$, then $R(A) \rightarrow 0$ and $Q(A) \rightarrow 0.1$, meaning that all MUs tend to have the same threshold and be instantaneously recruited. The muscle force is then close to $10 \%$ of the maximal muscle force since, at recruitment, each MU contracts at $10 \%$ of its tetanic force $(1-c=0.1)$. If $A \rightarrow 0$, then $R(A) \rightarrow+\infty$ and $Q(A) \rightarrow 0.66$. The muscle force increases with a slope close to 0 . Each recruited MU increases its firing frequency nearly to the tetanic contraction force until the next is recruited. Thus frequency modulation and recruitment develop parallel to each other, and at completion of the MU recruitment, the muscle force approaches $66 \%$ of its maximal force.

Relative activation surface. We know that $\mathcal{F}($ In $)=\int_{t_{\min }}^{t_{\max }} \rho(s) f(s$, In $) d s$, where $f(s, I n)=s\left(1-c \exp \left(-\alpha \frac{\operatorname{In-In_{T}(s)}}{\operatorname{In}\left(n_{T}(s)\right.}\right)\right)$ if $\operatorname{In}>\operatorname{In} n_{T}(s)$ and 0 otherwise. According to (7) and (21), we have

$$
\tilde{I} n_{T}(s)=F_{(1,1)}\left(\frac{\tilde{H}(s)}{A}\right)+1, \quad s \in\left[t_{\text {min }}, t_{\text {max }}\right] .
$$

By using the preceding relations and the change of variable $u=\bar{H}(s)$, for $\tilde{I} n \geq 1$, we get

$$
\tilde{\mathcal{F}}(\tilde{I} n)=\int_{0}^{1} I_{\left\{u \leq A F_{(1,1)}^{-1}(\tilde{I} n-1)\right\}}\left(1-c \exp \left(-\alpha \frac{\tilde{I} n-\left(F_{(1,1)}\left(\frac{u}{A}\right)+1\right)}{F_{(1,1)}\left(\frac{u}{A}\right)+1}\right)\right) d u
$$

$I_{E}$ denoting the indicator function of the set $E$. For fixed $\alpha$ and $c$, we see that $\tilde{\mathcal{F}}(\tilde{I} n)$ is a function of $\tilde{I} n$ and $A$ only. Therefore, it can be interpreted as a surface $\Sigma$, called the relative activation surface (Figure 4 ) and denoted $\tilde{\mathcal{F}}(A, \tilde{I} n)$. 


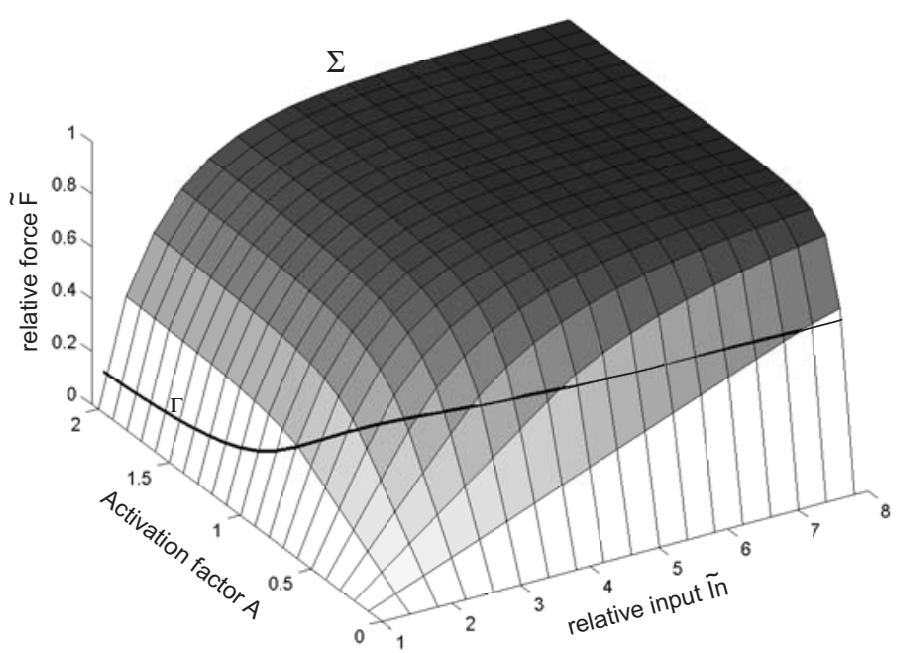

FIG. 4. The relative activation surface, the relation between the relative input, the activation factor, and the relative force. Vertical sections parallel to the $(\tilde{F}, \tilde{I} n)$ planes are the relative activation curves. The interconnected ends of the relative activation curves give the recruitment boundary curve (bold line).

Since the function $\hat{F}_{(1,1)}$ is strictly increasing, (24) implies the equivalence of $A_{1}<A_{2}$ and $\tilde{\mathcal{F}}\left(A_{1}, \tilde{I} n\right)<\tilde{\mathcal{F}}\left(A_{2}, \tilde{I} n\right)$ for all $\tilde{I} n>1$. Consequently, their projections on the (input, force) plane (Figure 3A) never intersect each other for $\tilde{I} n>1$. The end of the affine part of an activation curve is a point on $\Sigma$ corresponding to the end of recruitment. The set of these points defines a curve $\Gamma$ called the recruitment boundary curve (bold line in Figure 4). One of the parametric forms of $\Gamma$ is $0<A \mapsto$ $(A, 1+R(A), Q(A))$. We can project $\Gamma$ on three different planes:

(a) The projection on $(A, \tilde{\mathcal{F}})$ provides the recruitment ratio $Q(A)$ (Figure $3 \mathrm{C}$ ).

(b) The projection on $(A, \tilde{I} n)$ provides, up to a translation, the relative recruitment range $R(A)$ (Figure 3B).

(c) The projection on $(\tilde{I} n, \tilde{\mathcal{F}})$ provides the end-recruitment curve. Simple computations lead to its representation $\tilde{I} n \mapsto \frac{\tilde{I} n-1}{F_{(1,1)}^{-1}(\tilde{I} n-1)}$ (Figure 3A), which, according to Theorem $12(\mathrm{e})$, is an increasing function.

The vertical sections of $\Sigma$ parallel to the $(\tilde{I} n, \tilde{\mathcal{F}})$ plane are the relative activation curves, and according to (24), each one of them is determined by $A$ only (Figure 3A).

Relative force contributions due to recruitment and frequency modulation and related functions. The total muscle force is the sum of the contributions due to recruitment and frequency modulation $\mathcal{F}(\tilde{I} n)=\mathcal{F}^{\text {rec }}(\tilde{I} n)+\mathcal{F}^{\text {mod }}(\tilde{I} n)$. Dividing by $F_{\text {max }}$, we get $\tilde{\mathcal{F}}(\tilde{I} n)=\tilde{\mathcal{F}}^{\text {rec }}(\tilde{I} n)+\tilde{\mathcal{F}}^{\text {mod }}(\tilde{I} n)$. It is sufficient to compute $\tilde{\mathcal{F}}^{r e c}$, which is the fraction $(1-c)$ of the maximal force produced by all recruited MUs for the input $\tilde{I} n$. Thus $\tilde{\mathcal{F}}^{r e c}(\tilde{I} n)=(1-c) \frac{H(t(\tilde{I})))}{F_{\max }}$, where $t(\tilde{I} n)$ is the tetanic force of the strongest MU recruited by the relative input $\tilde{I} n$. By using (23), we get $\tilde{I} n=F_{(1,1)}\left(\frac{H(t(\tilde{I} n))}{\Delta}\right)+1$, and hence,

$$
\tilde{\mathcal{F}}^{r e c}(\tilde{I} n)= \begin{cases}(1-c) A F_{(1,1)}^{-1}(\tilde{I} n-1) & \text { if } 1 \leq \tilde{I} n \leq \tilde{I} n\left(t_{\max }\right), \\ 1-c & \text { if } \tilde{I} n>\tilde{I} n\left(t_{\max }\right) .\end{cases}
$$


Again we see that the relative muscle forces due to recruitment and to frequency modulation, as functions of $\tilde{I} n$, depend only on the activation factor. Straightforward computations provide the unit independent quotients for $1 \leq \tilde{I} n \leq \operatorname{In}_{T}\left(t_{\max }\right)$ :

$$
\begin{gathered}
\frac{\mathcal{F}^{r e c}}{\mathcal{F}}(\tilde{I} n)=\frac{\tilde{\mathcal{F}}^{r e c}}{\tilde{\mathcal{F}}}(\tilde{I} n)=(1-c) \frac{F_{(1,1)}^{-1}(\tilde{I} n-1)}{\tilde{I} n-1}, \\
\frac{\mathcal{F}^{\text {mod }}}{\mathcal{F}^{r e c}}(\tilde{I} n)=\frac{\tilde{\mathcal{F}}^{\text {mod }}}{\tilde{\mathcal{F}}^{r e c}}(\tilde{I} n)=\frac{1}{1-c} \frac{\tilde{I} n-1}{F_{(1,1)}^{-1}(\tilde{I} n-1)}-1 .
\end{gathered}
$$

We call the recruitment rate the number $100 \frac{\tilde{\mathcal{F}}^{\text {rec }}}{\tilde{\mathcal{F}}}$ (Figure $2 \mathrm{C}$ ) since it gives, at the end of recruitment, the percentage of the force due to recruitment. The ratio $\frac{\tilde{\mathcal{F}}^{\text {mod }}}{\mathcal{F}_{\text {rec }}}$ will be called frequency modulation to recruitment ratio or simply FMR (Figure 2B).

According to Theorem $12(\mathrm{e}), \frac{\tilde{\mathcal{F}}^{\text {rec }}}{\tilde{\mathcal{F}}}$ is decreasing and $\frac{\tilde{\mathcal{F}}^{\text {mod }}}{\tilde{\mathcal{F}}^{\text {rec }}}$ increasing. Both functions are muscle-independent as long as recruitment is not achieved. At the end of recruitment (e.r.), we have $\tilde{I} n-1=R(A)$ and thus

$$
\left.\frac{\mathcal{F}^{\text {rec }}}{\mathcal{F}}\right|_{\text {e.r. }}=\left.\frac{\tilde{\mathcal{F}}^{\text {rec }}}{\tilde{\mathcal{F}}}\right|_{\text {e.r. }}=\frac{(1-c)}{Q(A)},\left.\quad \frac{\mathcal{F}^{\text {mod }}}{\mathcal{F}^{\text {rec }}}\right|_{\text {e.r. }}=\left.\frac{\tilde{\mathcal{F}}^{\text {mod }}}{\tilde{\mathcal{F}}^{\text {rec }}}\right|_{\text {e.r. }}=\frac{1}{1-c} Q(A)-1 .
$$

The last two quantities depend only on $A$, and according to Theorem 12(f), the first one is increasing and the second one is decreasing. For fixed values of $\Delta$, the same properties hold for the variable $F_{\max }$ instead of $A$.

Recruitment gain. The recruitment gain, introduced in [28] in the context of $\mathrm{H}$ reflexes, is the "size of threshold differences to recruit additional MUs." The situation is simple in the case of the H-reflex since MUs are activated only once during this reflex and rate modulation is thus nonexistent. The recruitment gain corresponds to the derivative, during recruitment, of the number of active MUs with respect to the relative input. It is given by $R g(\tilde{I} n)=\frac{d}{d \tilde{I} n} \int_{t_{m i n}}^{t(\tilde{I} n)} \rho(s) d s=\rho(t(\tilde{I} n)) \frac{d}{d \tilde{I} n} t(\tilde{I} n)$. As we have seen before, $t(\tilde{I} n)=H^{-1}\left(\Delta F_{(1,1)}^{-1}(\tilde{I} n-1)\right)$, and by differentiating both sides with respect to $\tilde{I} n$ and denoting ${ }^{\prime}=\frac{d}{d \tilde{I} n}$, we obtain $\rho(t(\tilde{I} n)) t(\tilde{I} n) t^{\prime}(\tilde{I} n)=$ $\Delta\left(F_{(1,1)}^{-1}\right)^{\prime}(\tilde{I} n-1)$. Finally, the last three equations lead to

$$
R g(\tilde{I} n)=\frac{\Delta\left(F_{(1,1)}^{-1}\right)^{\prime}(\tilde{I} n-1)}{H^{-1}\left(\Delta F_{(1,1)}^{-1}(\tilde{I} n-1)\right)} .
$$

In contrast to the preceding relations derived in this section, $R g(\tilde{I} n)$ depends on the particular muscle via $H$ and $\Delta$.

5. Discussion. The relative synaptic weight, which specifies the efficacy of the synaptic input to the individual MNs, cannot be determined with the present experimental techniques. However, every model of the MNPMC requires this quantity, and we present here an approach allowing its computation. A main feature of the model is that it is based on a known behavior of the activation curve during recruitment. Indirect measurements [38] indicate that this function is affine during recruitment, and this turns out to be sufficient to determine the relative synaptic weight. The MNPMC model can now be used to compute various functionals related to the muscle activation. They provide a deeper insight into the processes occurring during muscle activation 
and also yield values for missing experimental data required for more complex models of the MNPMC. The computed relative synaptic weight has been implemented in a time-dependent model which considers each MU individually [36, 39].

Normalizing the force by the maximal muscle force $F_{\max }$ and the input by the threshold input $I n_{0}$ is the source of several advantages. In this new frame, the slope $k$ of the activation curve during recruitment is transformed into the activation factor $A=\frac{k I n_{0}}{F_{\max }}$. The signature in $A$ of the muscle MU population is $F_{\max }$, the first moment of $\rho$. One could have expected a more intricate dependence since $\rho$ is an arbitrary nonnegative and integrable function.

The activation factor is the parameter which governs completely the activation process of a muscle in our model. Indeed, the relative activation surface depends on $\tilde{I} n$ and $A$. It turns out that the relative activation curve depends only on $A$ (even after recruitment is completed), entailing that the recruitment boundary curve $\Gamma$, the end-recruitment curve $\gamma$, the relative activation curves, the relative recruitment ratio $Q$, and the relative recruitment range $R(A)$ depend only on $A$.

It is also quite remarkable that the ratios $\frac{\tilde{\mathcal{F}}^{\text {mod }}}{\mathcal{F}^{\text {rec }}}=\frac{\mathcal{F}^{\text {mod }}}{\mathcal{F}^{\text {rec }}}$ and $\frac{\tilde{\mathcal{F}}^{\text {rec }}}{\mathcal{\mathcal { F }}}=\frac{\mathcal{F}^{\text {rec }}}{\mathcal{F}}$, as long as recruitment is not achieved, depend only on the function $F_{(1,1)}^{-1}$ and are thus totally independent of the muscle. Of course, the values of these ratios depend on $A$ at the end of recruitment. However, the relative synaptic weight depends on $I n_{0}$ and $A$, and finally, the recruitment gain $R g(\tilde{I} n)$ depends on $\Delta$ and $\rho$. As in [45], several functionals become muscle-independent or depend only on the activation factor.

The preceding normalization also allows for the comparison of muscles with different strengths, as described in [42] for the first dorsal interosseus, a small hand muscle, and the gastrocnemius, a much stronger leg muscle.

We proved that for fixed values of $k$ and $I n_{0}$, the MU population density $\rho$ and the relative synaptic weight $\tilde{g}$ are linked by a one-to-one relation. The MU population $\rho$ of a muscle can therefore be recovered from the synaptic weight and conversely is a feature which might be used by the CNS. If the properties of an MU population change by a lesion or a pathological situation such as muscular dystrophy, or simply by disuse or training, an input to the MN pool does not result in the force expected by the CNS. As a consequence, the relative synaptic weight in the MN pool might be readjusted in order to achieve the activation curve required for a properly working motor control. Sensory input from muscle spindles and additional sensors might play a major role in such a feedback system. This hypothesis could be tested in patients with motor diseases, in subjects participating at bed rest and thus concerned with muscle atrophy, or in subjects undergoing a force training.

The activation curve is composed of an affine part, prescribed a priori during recruitment, followed by a nonaffine portion due to frequency modulation only. Our model predicts that the affine part can be maintained up to at most $66 \%$ of the maximal muscle force, a situation achievable with a slope approaching 0 . For muscle forces above the relative recruitment range, the slope of the activation curve is steadily decreasing. As a consequence, relatively strong inputs are required to adjust high force levels. The activation curve has not yet been investigated in muscles with a large activation factor where the nonaffine range extends over an important domain of the input. However, there is evidence that an affine relationship holds in the working range of the human soleus muscle. Unpublished data (D. G. Ruegg and T. H. Kakebeeke) show that humans are able to voluntarily contract the soleus muscle up to only about $60 \%$ of its maximal force, and the activation curve is affine over that range, suggesting that the behavior of the soleus is compatible with our model. 
In this way, the CNS might limit the muscle's working field to the affine range, a possibly very useful property. Indeed, motor centers that are hierarchically above the MN pool would be faced with a fixed activation curve. Consequently, a control mechanism at the spinal level would be sufficient to adjust the synaptic weight in order to maintain the activation curve, when changes in the MU population are induced by training, disuse, or disease. Moreover, the whole activation curve is automatically adjusted by the affine part since it depends only on its slope, namely, the activation factor. The verification of this property requires subjects with a modified MU density function.

\section{REFERENCES}

[1] J. ACZEL, Vorlesungen über Funktionalgleichungen und ihre Anwendungen, Birkhäuser Verlag, Basel, 1961.

[2] F. Baldissera and B. Gustafsson, Firing behavior of a neurone model based on the afterhyperpolarization conductance time course. First interval firing, Acta Physiol. Scand., 91 (1974), pp. 528-544.

[3] F. D. Bremmer, J. R. Baker, and J. A. Stephens, Correlation between the discharges of motor units recorded from the same and from different finger muscles in man, J. Physiol. Lond., 432 (1991), pp. 355-380.

[4] H. Brunner and P. J. Van der Houwen, The Numerical Solution of Volterra Equations, North-Holland, Amsterdam, 1986.

[5] W. H. Calvin And P. C. Schwindt, Steps in production of motoneuron spikes during rhythmic firing, J. Physiol., 35 (1972), pp. 297-310.

[6] K. L. Chung, A Course in Probability Theory, Harcourt, Brace and World, New York, 1968.

[7] A. K. Datta And J. A. Stephens, Synchronization of motor unit activity during voluntary contraction in man, J. Physiol. Lond., 422 (1990), pp. 397-419.

[8] J. E. Desmedt And E. Godaux, Ballistic contractions in man: Characteristic recruitment pattern of single motor units of the tibialis anterior muscle, J. Physiol. Lond., 264 (1977), pp. 673-693.

[9] J. E. Desmedt and E. Godaux, Fast motor units are not preferentially activated in rapid voluntary contractions in man, Nature Lond., 267 (1977), pp. 717-719.

[10] T. M. Flett, Mathematical Analysis, McGraw-Hill, New York, 1966.

[11] O. Forster, Analysis 2, Friedr. Vieweg \& Sohn, Braunschweig, Wiesbaden, Germany, 1996.

[12] A. J. Fuglevand, K. M. Zackowski, K. A. Huey, And R. M. EnokA, Impairment of neuromuscular propagation during human fatiguing contractions at submaximal forces, J. Physiol. Lond., 460 (1993), pp. 549-572.

[13] R. A. F. Garnett, M. J. O'Donovan, J. A. Stephens, and A. Taylor, Motor unit organization of human medial gastrocnemius, J. Physiol. Lond., 287 (1978), pp. 33-43.

[14] G. Gripenberg, S. O. Londen, and O. Staffans, Volterra Integral and Functional Equations, Cambridge University Press, Cambridge, UK, 1990.

[15] P. J. HARRISON AND A. TAYLOR, Individual excitatory post-synaptic potentials due to muscle spindle I $a$ afferents in cat triceps surae motoneurones, J. Physiol., 312 (1981), pp. 455-470.

[16] C. J. Heckman and M. D. Binder, Analysis of effective synaptic currents generated by homonymous I a afferent fibers in motoneurons of the cat, J. Neurophysiol., 60 (1988), pp. 1946-1966.

[17] C. J. Heckman And M. D. Binder, Computer simulation of the steady-state input-output function of the cat medial gastrocnemius motoneuron pool, J. Neurophysiol., 65 (1991), pp. 952-967.

[18] C. J. Heckman and M. D. Binder, Computer simulations of motoneuron firing rate modulation, J. Neurophysiol., 69 (1993), pp. 1005-1008.

[19] C. J. Heckman and M. D. Binder, Computer simulations of the effects of different synaptic input systems on motor unit recruitment, J. Neurophysiol., 70 (1993), pp. 1827-1840.

[20] C. J. HeCKMAn AND M. D. Binder, Computer simulations of the effects of different synaptic input systems on the steady-state input-output structure of the motoneuron pool, J. Neurophysiol., 71 (1994), pp. 1727-1739.

[21] E. Henneman, Principles governing distribution of sensory input to motor neurons, in The Neurosciences: Third Study Program, F. O. Schmitt and F. G. Worden, eds., MIT Press, Cambridge, MA, 1974, pp. 281-291. 
[22] A. L. Hodgkin And A. F. Huxley, A quantitative description of membrane current and its application to conduction and excitation in nerve, J. Physiol., 117 (1952), pp. 50-544.

[23] K. Kanosue, M. Yoshida, K. Akazawa, And K. FujiI, The number of active motor units and their firing rates in voluntary contraction of human brachialis muscle, Jpn. J. Physiol. Lond., 29 (1979), pp. 427-443.

[24] D. KernelL, The limits of firing frequency in cat lumbosacral motoneurones possessing different time course of afterhyperpolarization, Acta Physiol. Scand., 65 (1965), pp. 87-100.

[25] D. Kernell, High-frequency repetitive firing of cat lumbosacral motoneurones stimulated by long-lasting injected currents, Acta Physiol. Scand., 65 (1965), pp. 74-86.

[26] D. KeRnell, Functional properties of spinal motoneurons and graduation of muscle force, in Motor Control Mechanisms in Health and Disease, J. E. Desmedt, ed., Raven Press, New York, 1983, pp. 213-226.

[27] D. KERNELL, Rhythmic properties of motoneurones innervating muscle fibres of different speed in m. gastrocnemius medialis of the cat, Brain Res., 160 (1979), pp. 159-162.

[28] D. Kernell And H. Hultborn, Synaptic effects on recruitment gain: A mechanism of importance for the input-output relations of motoneurone pools?, Brain Res., 507 (1990), pp. $176-179$.

[29] M. Kuczma, An introduction to the theory of functional equations and inequalities, in Panstwowe Wydawnictwo Naukowe, Uniwersytet Slaski, Warzawa, 1985, pp. 424-426.

[30] C. G. Kukulka and P. Clamann, Comparison of the recruitment and discharge properties of motor units in human brachial biceps and adductor pollicis during isometric contractions, Brain Res., 219 (1981), pp. 45-55.

[31] G. W. H. Mantel and R. N. Lemon, Cross-correlation reveals facilitation of single motor units in thenar muscles by single corticospinal neurons in the conscious monkey, Neurosci. Lett., 77 (1987), pp. 113-118.

[32] R. K. Miller, Nonlinear Volterra Integral Equations, W. A. Benjamin, Menlo Park, CA, 1971.

[33] H. S. Milner-Brown, R. B. Stein, And R. Yemm, The orderly recruitment of human motor units during voluntary isometric contractions, J. Physiol. Lond., 230 (1973), pp. 359-370.

[34] H. S. Milner-Brown, R. B. Stein, And R. Yemm, Changes in firing rate of human motor units during linearly changing voluntary contractions, J. Physiol. Lond., 230 (1973), pp. 371-390.

[35] A. W. Monster And H. Chan, Isometric force production by motor units of extensor digitorum communis muscle in man, J. Neurophysiol., 40 (1977), pp. 1432-1443.

[36] R. M. Nussbaumer, D. G. RüegG, L. M. Studer, and J.-P. Gabriel, Computer simulation of the motoneuron pool-muscle complex. I. Input system and motoneuron pool, Biol. Cybern., 86 (2002), pp. 317-333.

[37] S. S. PALmer AND E. E. Fetz, Effects of single intracortical microstimuli in motor cortex on activity of identified forearm motor units in behaving monkeys, J. Neurophysiol., 54 (1985), pp. 1194-1212.

[38] D. G. Ruegg and F. Bongioanni, Superposition of ballistic on steady contractions in man, Exp. Brain Res., 77 (1989), pp. 412-420.

[39] M.-A. Schnetzer, D. G. RuegG, R. Baltensperger, and J.-P. Gabriel, Three-dimensional model of the muscle structure and the surface EMG, in Proceedings of the 5th European Conference of the European Society of Mathematical and Theoretical Biology (ESMTB) on Mathematical Modelling \& Computing in Biology and Medicine (Milan, Italy, 2002), Esculapio, Bologna, Italy, 2003.

[40] P. J. Slot And T. SinkJAeR, Simulations of the alpha motoneuron pool electromyogram reflex at different preactivation levels in man, Biol. Cybern., 70 (1994), pp. 351-358.

[41] D. R. Smart, Fixed Point Theorems, Cambridge University Press, Cambridge, UK, 1974.

[42] L. M. Studer, D. G. Ruegg, and J. P. Gabriel, A model for steady isometric muscle activation, Biol. Cybern., 80 (1999), pp. 339-355.

[43] A. A. M. TAX AND J. J. Denier van DER Gon, A model for neural control of gradation of muscle force, Biol. Cybern., 65 (1991), pp. 227-234.

[44] R. J. H. Wilmink And P. J. SLOt, Modeling of the H-reflex facilitation during ramp and hold contractions, J. Comput. Neurosci., 3 (1996), pp. 337-346.

[45] F. E. ZAJAC, Muscle and tendon: Properties, models, scaling, and application to biomechanics and motor control, Crit. Rev. Biomed. Eng., 17 (1989), pp. 359-411. 